

\title{
COMPOSITION EN VITAMINE A ET EN CAROTĖNE DE BEURRES DE CONSOMMATION DE L'ÉTAT DE SAO PAULO (BRÉSIL)
}

P. Mucciolo, O. Barbuto, M. M. Campos

\section{- To cite this version:}

P. Mucciolo, O. Barbuto, M. M. Campos. COMPOSITION EN VITAMINE A ET EN CAROTĖNE DE BEURRES DE CONSOMMATION DE L'ÉTAT DE SAO PAULO (BRÉSIL). Le Lait, 1956, 36 (358), pp.494-500. hal-00928174

\section{HAL Id: hal-00928174 \\ https://hal.science/hal-00928174}

Submitted on 1 Jan 1956

HAL is a multi-disciplinary open access archive for the deposit and dissemination of scientific research documents, whether they are published or not. The documents may come from teaching and research institutions in France or abroad, or from public or private research centers.
L'archive ouverte pluridisciplinaire HAL, est destinée au dépôt et à la diffusion de documents scientifiques de niveau recherche, publiés ou non, émanant des établissements d'enseignement et de recherche français ou étrangers, des laboratoires publics ou privés. 


\title{
BIBLIOGRAPHIE
}

[1] P. Schain. Science, 1949, 110, 121.

[2] L. Gershenfeld and B. I. Ucko. Milk and food Technology, 1950, 13, 175.

[3] J. Pien. "Vues actuelles sur le dosage de la matière grasse du lait ", dáns Mises au point de chimie analytique pure et appliquée et d'analyses bromatologiques, publiées sous la direction de J. A. Gadtier, $3^{\text {e }}$ série, 1 volume de 192 pages, Masson éditeur, 120, Boulevard SaintGermain, Paris $\left(6^{\mathrm{e}}\right) .1955$.

[4] F. Aftalion. Les polyoxyéthylèneglycols en cosmétologie. La Parfumerie moderne, 1953, no 34 , juillet-août.

[5] J. P. Sisley. Index des huiles sulfonées et détergents modernes, p. 535. Paris, 1949.

\section{COMPOSITION EN VITAMINE A \\ ET EN CAROTËNE DE BEURRES DE CONSOMIMATION DE L'ÉTAT DE SAO PAULO (BRÉSIL)}

\author{
par
Professeur P. MUCCIOLO, O. BARBUTO, M. M. CAMPOS
Directeur
Technologiste
Chimiste

Laboratoire du "Département de l'Industrie, inspection et conservation des produits alimentaires d'origine animale», de la Faculté de Médecine Vétérinaire de l'Université de Sao Paulo (Brésil)

Notre but, en entreprenant ce travail, était de connaître la composition en vitamine $\mathrm{A}$ et en carotène des beurres fabriqués dans l'Etat de Sao Paulo. Des enquêtes de cette catégorie ont déjà été réalisées dans de nombreux pays, et il n'est pas nécessaire d'insister sur leur importance pratique. Dans l'Etat de Sao Paulo, nous n'avons trouvé, comme étude semblable, qu'une seule étude, de Cecy Mello Texeira [1] présentant les moyennes de 61 analyses effectuées sur des beurres recueillis au marché de la ville de Sao Paulo.

Désirant étendre cette enquête, et surtout observer les variations de composition dues aux époques différentes de l'année, nous avons eu de grosses difficultés relativement à l'obtention des échantillons en temps voulu, ce qui nous a privé des éléments nécessaires pour pouvoir en toute sécurité présenter l'analyse statistique de nos résultats. Dans les conditions où nous avons opéré, nous ne pouvons prétendre que nos résultats peuvent être pris comme règle générale, malgré la quantité appréciable de matériel analysé, provenant de presque tous les centres importants de production de beurre de l'Etat de Sao Paulo, dont quelques-uns de l'Etat du Minas Gérais qui fournissent également la ville de Sao Paulo. 
Nos analyses se réfèrent done à de nombreux échantillons de beurres de consommation de l'Etat de Sao Paulo, mais les résultats ne peuvent être interprétés comme ceux de la production générale de l'Etat.

\section{Matériel et méthodes}

Nous avons analysé 189 échantillons de beurres, dont la répártition mensuelle est marquée dans le tableau I, le long d'une période qui s'étend de juin 1954 à mai 1955, et dont l'origine a du être cataloguée arbitrairement, suivant la distribution :

Zone I : Paulo de Faria, Nova Granada, Olimpia, Ibirá, Sao José do Rio Preto, Poloni, Bebedouro, Pitangueiras, Guaira, Pirangi, Orlandia, Ibitinga e Araraquara. Zone II : Avaí, Duartina, Araras, Laranjal, Jundiaí. Zone III : Mocóca, Sao José do Rio Pardo, Itobí, Guaxupé, Sao Gonçalo do Sapucaí. Zone IV : Vale do Paraíba (Sao José do Campos, Taubaté, Pindamonhangaba, Cachoeira Paulista, Barreiro), Paraísópolis. Zone $V$ : Cerqueira Cesar, Angatuba, Tatuí. Zone VI : Assis.

Malheureusement, là aussi, malgré la diversité de provenance des échantillons, leur nombre n'est pas suffisamment élevé pour conclure par une expression statistiqué du résultat de nos anaJyses.

L'extraction de la vitamine $\mathrm{A}$ et du carotène a été réalisée d'après la technique de McDow EL [2] qui consiste en une saponification ménagée, suivie d'extractions par l'éther privé de péroxydes, et distillation finale de l'extrait à $60^{\circ}$ sous courant de $\mathrm{CO}^{2}$. On dissout le résidu dans du chloroforme purifié.

La vitamine A est dosée par le réactif de CARR-PRICE, à $620 \mu$, à l'aide d'un spectrophotomètre Coleman, et en utilisant comme référence une solution d'acétate de vitamine A U.S.P.

Le carotène est dosé sur une partie aliquote à l'aide du même spectrophotomètre, à la longueur d'onde de $440 \mu$, en utilisant comme étalon, une solution de $\beta$ carotène pur d'origine française. Il est nécessaire de soustraire de la couleur totale du dosage du carotène la part due aux xantophylles. Nous avons déterminé celle-ci par la méthode de Berl-Peterson, légèrement modifiée, sur quarante échantillons, suivant en cela l'exemple donné par plusieurs auteurs [3]. Pour cause de difficulté matérielle, nous n'avons pu étendre le dosage des xantophylles à tous les échantillons, mais la constance de la teneur en celles-ci, qui est de $14 \%$ de la coloration lors du dosage du carotène, nous a fait prendre ce chiffre pour la correction des dosages du carotène dans tous les échantillons. Dans nos dosages, nous admettons done que $86 \%$ de la coloration rouge qui se développe est due au carotène. 


\section{Résultats}

Les résultats de nos analyses sont exprimés dans le tableau I, les teneurs de carotène y sont inscrites en microgrammes, celles de vitamine A en unités internationales, ainsi que celles de vitamine A totale. Toutes les teneurs se rapportent à 1 gramme de matière grasse de beurre. Nous signalons ce détail, car comme il n'existe pas de normalisation à cet égard, les auteurs donnent parfois leurs résultats sur poids de beurre, d'autres omettent de signaler si leurs analyses se rapportent à la matière grasse du beurre, ou simplement au poids total de celui-ci.

Dans la colonne réservée à la vitamine A totale, dans le tableau I, nous exprimons sous cette dénomination la somme, vitamine A en unités internationales, et la valeur du carotène présent, calculé également en unités internationales, -car comme le carotène du

TABLEAU I

MOYENNES MENSUELLES DE GAROTENE, VITAMINE A ET VITAMINE A TOTALE

\begin{tabular}{|c|c|c|c|c|}
\hline Mois & $\begin{array}{c}\mathrm{N}^{\circ} \mathrm{de} \\
\text { l'échantillon }^{-}\end{array}$ & $\begin{array}{c}\text { Carotène } \\
\mu \text { g par gr. de } \\
\text { matière grasse }\end{array}$ & $\begin{array}{l}\text { Vitamine A } \\
\text { U.I. par gr. de } \\
\text { matière grasse }\end{array}$ & $\begin{array}{l}\text { Vitamine A tot. } \\
\text { U.I. par gr. de } \\
\text { matière grasse }\end{array}$ \\
\hline Juin $1954 \ldots \ldots$ & 9 & 5,5 & 29,2 & 38,4 \\
\hline Juillet $\ldots \ldots \ldots$ & 14 & 6,3 & 30,8 & 41,3 \\
\hline Août $\ldots \ldots \ldots$ & 14 & 5,6 & 33,1 & 42,4 \\
\hline Septembre .... & 15 & 5,2 & 30,9 & 39,7 \\
\hline Octobre ...... & 12 & 6,1 & 35,6 & 45,9 \\
\hline Novembre.... & 21 & 7,1 & 28,7 & 40,6 \\
\hline Décembre .... & 19 & 7,3 & 26,8 & 39,0 \\
\hline Janvier $1955 \ldots$ & 18 & 7,2 & 25,7 & 37,7 \\
\hline Février $\ldots \ldots \ldots$ & 11 & 6,4 & 26,2 & 36,9 \\
\hline Mars $\ldots \ldots \ldots$ & 14 & 5,9 & 24,7 & 34,4 \\
\hline Avril $\ldots \ldots \ldots$ & 21 & 6,1 & 25,4 & 35,5 \\
\hline Mai .......... & 21 & 5,9 & 27,5 & 37,4 \\
\hline Total des échant. & 189 & & & \\
\hline Média anual ... & & 6,2 & 28,7 & 39,1 \\
\hline
\end{tabular}

beurre est transformé et utilisé en totalité par l'organisme, on peut par cette somme exprimer un potentiel vitaminique. Comme nous l'avons déjà dit, la difficulté d'obtenir de certains industriels un envoi régulier et suivi des échantillons de beurre, nous a empêché entre autre de présenter des résultats extensibles directement en ce qui concerne la production générale de l'Etat, mais encore, nous a fait renoncer à présenter séparément chaque analyse de beurre; 
nous ayons jugé préférable de grouper nos résultats en moyennes mensuelles et annuelles, donnant ainsi simplement un apperçu général de la composition vitaminique des beurres livrés à la consommation dans l'Etat de Sao Paulo. Comme le montre le tableau I, la moyenne annuelle de nos analyses de 189 échantillons, allant de juin 1954 à mai 1955, donne 6,2 microgrammes de carotène, 28,7 U.I. de vitamine A, et 39,1 U.I. de vitamine A totale, par gramme de matière grasse de beurre.

\section{Discussion}

La publication de Mello Texeira [1] qui porte sur l'analyse de 61 échantillons recueillis sur le marché de Sao Paulo, n'indique pas si les résultats se rapportent à la vitamine A totale (ce qui paraît peu probable, étant donné que la détermination du carotène n'a pas été faite par cet auteur). De plus, l'auteur n'indique pas non plus la date et l'origine des échantillons prélevés, ce qui ne permet pas de procéder à une comparaison avec nos résultats.

La moyenne annuelle de vitamine A totale obtenue par nous ne diffère pas de beaucoup de celle rencontrée dans d'autres pays. Mais, pour toute comparaison valable à ce sujet, il faut insister sur le fait que les différences des teneurs en vitamine A des beurres sont dues surtout aux époques de l'année, comme le démontrent plusieurs auteurs [5], [6], [7], [8], [9], en relation directe avec la quantité et la qualité de l'alimentation du bétail. A titre d'indication, nous citons dans le tableau II, quelques données de la littérature spécialisée, qui se rapportent aux teneurs de vitamine A pendant les deux principales saisons de l'année.

TABLEAU II

MOYENNES ANNUELLES DE VITAMINE A TOTALE, D'APRES LES SAISONS A L'ÉTRANGER.

\section{Pays - Auteur}

Allemagne (Mohr) $(10) \ldots \ldots \ldots \ldots \ldots$.

Etats-Unis (Agrieult. Dep. (11) ......

Europe (Neri) (12) . . . . . . . . . .

Japon (Nakanishi) (13) . . . . . . . .

Nouvelle-Zélande (Barnicoat) (5) ....

En U.I. par gr. de matière grasse du beurre

$\begin{array}{ll}\text { Eté } & \text { Hiver } \\ 60 & 30 \\ 39,7 & 24,7 \\ 26 & 11 \\ 23,2 & 14,3 \\ 33-37 & 45-53\end{array}$

Dans son étude, Sarkar [6] reconnaît lui aussi, que dans l'Inde, le potentiel maximum en vitamine $\mathrm{A}$ du beurre se situe en été, et le minimum en hiver, de même qu'en Suède, où les recherches effectuées par l'Institut de la Santé pendant plusieurs années révèlent que la teneur en vitamine A pendant l'hiver et pendant l'été sont dans le rapport de 2,3. Observation identique a été faite par 
LoRD [14] en Angleterre. L'examen du tableau II montre aussi que le beurre de la Nouvelle-Zélande fait exception apparente à cette règle, sa teneur maximum vitaminique se place en hiver, ainsi que l'ont établi Barnicoat [5] et NoRTh [7], lesquels font la remarque que le comportement de la richesse vitaminique du beurre est l'inverse dans les hémisphères nord et sud. Cette particularité se rencontre également dans nos résultats, comme le montre le tableau III, appuyant, jusqu'à un certain point, les allégations de l'auteur néozélandais, c'est-à-dire que la variation de la teneur vitaminique est en relation avec l'hémisphère correspondant.

Obtenant ainsi des valeurs plus fortes de vitamine A totale en hiver et au printemps, et les plus faibles en été et en octobre, nos résultats suivent ceux obtenus en Nouvelle-Zélande, et s'inversent avec ceux de l'Europe. Cependant, il convient de remarquer que la

TABLEAU III

VARIATIONS DES TENEURS EN CAROTÉNE, VITAMINE A ET VITAMINE A TOTALE

Carotene
gr. par gr. de
matière grasse

Printemps : octobre, novembre, décembre

Eté : janvier, février, mars ............

Automne : avril, mai, juin ..........

Hiver : juillet, août, septembre ........

\author{
Vitamine A U.I. \\ par gr. de \\ matière grasse
}

$$
6,8
$$$$
6,5
$$$$
5,8
$$

30,4

25,5

27,4

31,6

\author{
Vitamine A totale \\ U.I. par gr. de \\ matière grasse
}

teneur en carotène ne suit pas cette même influence, fait noté aussi par NoRTh [7] pour la Nouvelle-Zélande, qu'il attribue au fait de la forte proportion de vaches Jersey, et que les troupeaux ont accès aux pâturages pendant toutes les périodes de l'année.

Etroitement liéé à la période saisonnière, la précipitation pluviométrique a une influence sensible sur les teneurs en vitamine A et carotène des beurres. Nos résultats montrent par la courbe du graphique I que les valeurs maxima en vitamine A total correspondent aux plus faibles chutes d'eau dans tout le territoire étudié, à l'exception du mois d'octobre.

\section{Résumé et conclusion}

Le but de ce travail a été de tenter de déterminer la teneur vitaminique (carotène et vitamine $\mathrm{A}$ ) des beurres consommés dans l'Etat de Sao Paulo. Par suite de difficultés d'ordre matériel et 


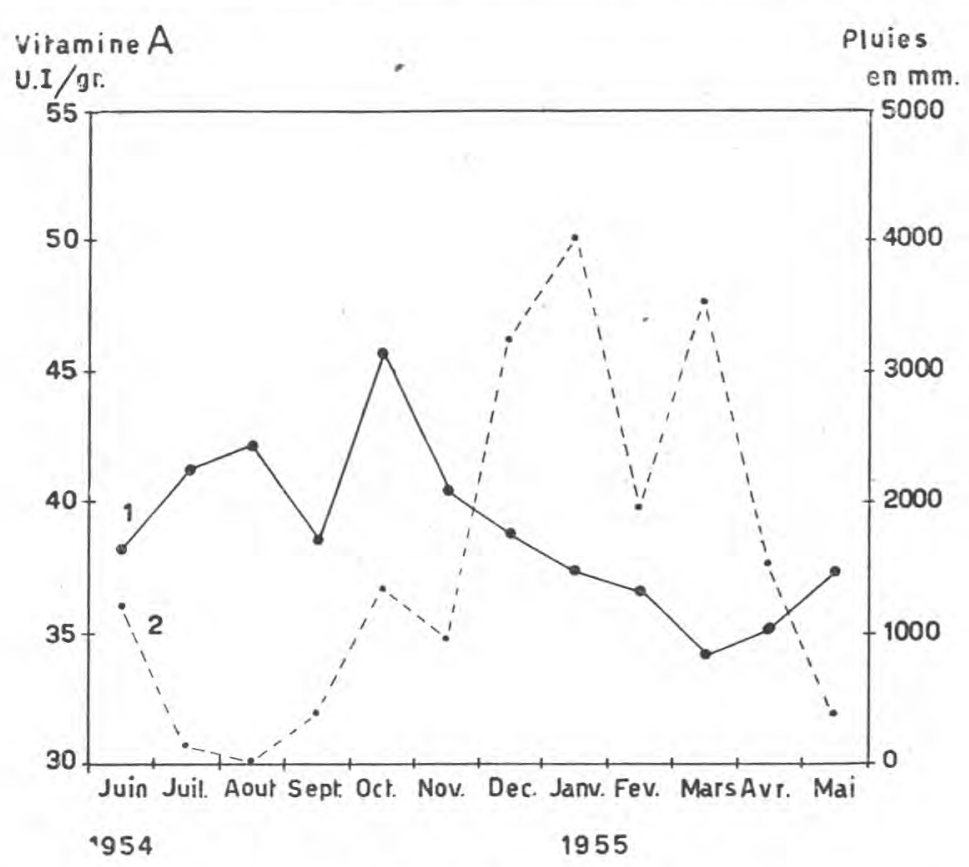

Graphique $n^{\circ} 1$

Relation entre la teneur en vitamine $A$ totale de quelques beurres de l'Etat de Sao Paulo et la pluviométrie.

(1) Teneur en vitamine A totale (carotène + vitamine A), en U.I. par gramme de matière grasse du beurre.

(2) Plúviométrie de l'ensemble de l'Etat de Sao Paulo.

malgré la variété des échantillons analysés, il a été impossible de procéder à l'analyse statistique des résultats, empêchant ainsi d'étendre ceux-ci au caractère général de la production.

Il a été analysé 189 échantillons de beurres produits dans les principaux centres de l'Etat de Sao Paulo, de la période de juin 1954 à mai 1955. Les teneurs en carotène, vitamine $\mathrm{A}$, ont été réalisés par la méthode de McDowes, utilisant le réactif de Carr-Price, à l'aide $\mathrm{du}$ spectrophotomètre Coleman Junior, et en faisant les lectures à $620 \mu$ pour la vitamine $\mathrm{A}$ et à $440 \mu$ pour le carotène. Il a été procédé aux dosages des xanthophylles sur $20 \%$ des échantillons, attribuant à celles-ci $14 \%$ de la valeur de la coloration totale lors du dosage du carotène, en suivant la technique de BerlPeterson modifiée dans les détails. Ce chiffre a été utilisé pour les corrections des dosages de carotène sur tous les échantillons. Dans les conditions de travail exposé, les auteurs concluent :

10 La moyenne des analyses de 189 échantillons de beurres de consommation recueillis dans l'Etat de Sao Paulo, dans la période 
de juin 1954 à mai 1955, a été de 6,2 microgrammes de carotène ; 28,7 U.I. de vitamine A et 39,1 U.I. de vitamine A totale, par gramme de matière grasse ;

$2^{\circ}$ Pour le carotène, la teneur maximum a été observée en février (11 microgrammes) et le minimum en octobre $(3,1$ microgrammes). Pour la vitamine A, la teneur maximum se situe en octobre (40,2 U.I.) et le minimum en décembre (20 U.I.). Pour la vitamine A totale, la richesse maximum a été observée en octobre (45,9 U.I.) et le minimum en mars (34,4 U.I.) ;

$3^{\circ}$ Les valeurs pour la vitamine A totale ont été observées, les plus fortes en hiver et printemps, les plus faibles en été et octobre ; $4^{\circ}$ Les valeurs maxima enregistrées pour la vitamine A totale, coïncident avec les plus faibles chutes de pluie de la région étudiée, mais à l'exception du mois d'octobre.

\section{BIBLIOGRAPHIE}

E. Akjerberg, V. Hellström. 1954. Milk and butter. Vitamin A content. Livsmedelsteknik. "in Food Manufacture, 30, 4, 163, 1955.

S. Bert, W. H. Peterson. 1943. Determinations and content of carotene and vitamin $\mathrm{A}$ in Wisconsin butter. Jour. Nutrition, 26, 527.

C. R. Bernicoat. 1947. Variations in the carotene and vitamin A contents of certain New Zealand butterfats. Jour. Dairy Res., 15, 80-88.

A. E. Gillam.1934. A modified spectrophotometrie method for the assay of carotene and vitamin A in butter. Biochem. Jour., 28, 79.

Institut de Recherches laitières Néo-zélandais. 1948-1949. Teneur en vitamine A du beurre de N.-Z. Rapp. annuel 1948-1949 in Le Lait, 31, $155-158,1951$.

J. W. LORD. 1945. Seasonal variation of carotene and vitamin A in butter-fat and in serum. Biochem. Jour., 39, 372-374.

A. K. R. McDoweLl. 1943. The estimation of vitamin A in butter. A critical study of methods. Jour. Dairy Res., 16, 348-355.

W. Монв. 1949. Structure and consistenct of butter. Suddent Molkerei Ztg., 70, 1289-1293, in Chem. Zentr., 1, 2299, 1950.

T. NAKANISHI. 1953. Studies on the improvement of butter making procedure. I. Vitamin A potency of Japanese butter. Tohoku Jour. Agric. Res., 4, 15-20, in Dairy Sci. Absts., 16, 11, 929, 1954.

A. Neri, 1955. Il burro. Il Latte, 29, 2, 103.

D. B. Parrish, W. H. Martin, F. W. Atkeson, J. S. Hughes. 1946. The vitamin A and carotene content of market butter produced in Kansas. Jour. Dairy Sai., 29, 2, 91-99.

B. C. R. SARKaR. 1948. Effect of season, breed and species of ruminants on the vitamin A potency of butterfat. Jour. Dairy Sci., 31, 3, 165-172.

C. Mello Tetxeira. 1950. Determinaçao de vitamina A em manteiga. An. la. Jornada Brasileira Bromatologia, S. Paulo, 3-13 avril, 1946, 2, 809-813.

U.S. Department of Agriculture. 1947. Le beurre comme source de vitamine A dans l'alimentation de la population des Etats-Unis. Publ. 636. in Le Lait, 29, 491.492, 1949. 